\title{
Is the Long Memory Factor Important for Extending the Fama and French Five-Factor Model: Evidence from China
}

\author{
Yicun Li, ${ }^{1}$ Yuanyang Teng, ${ }^{1}$ Wei Shi, ${ }^{1}$ and Lin Sun $\mathbb{D}^{2}$ \\ ${ }^{1}$ School of Management, Zhejiang University, Hangzhou, China \\ ${ }^{2}$ School of Applied Mathematics, Guangdong University of Technology, Guangzhou, China
}

Correspondence should be addressed to Lin Sun; yss112@gdut.edu.cn

Received 10 May 2021; Accepted 10 June 2021; Published 24 June 2021

Academic Editor: Wen-Tsao Pan

Copyright $@ 2021$ Yicun Li et al. This is an open access article distributed under the Creative Commons Attribution License, which permits unrestricted use, distribution, and reproduction in any medium, provided the original work is properly cited.

This paper proposes a new factor model, which is built upon the marriage of the Fama and French five-factor model and a long memory factor based on the monthly data of the A-share market in the Chinese stock market from January 2010 to July 2020 . We first examine the explanatory power of the Fama and French five-factor model. We find strong market factor return of market $(\mathrm{RM})$, size factor small minus big (SMB), and value factor high minus low (HML) but weak factor robust minus weak (RMW) and investment factor conservative minus aggressive (CMA). Then, both the Hurst exponent and the momentum factors (MOM) are added to the model to test the improvement of the explanatory power of these two new factors. We find that both the momentum factor and the Hurst exponent factor can effectively improve the explanatory power of the model. The momentum factor captures the short-term trend, but it cannot completely replace the Hurst exponent, which reflects the long memory effect.

\section{Introduction}

In the field of quantitative investment, factor models have always attracted much attention. In 1993, Fama and French proposed a celebrated three-factor model including a size factor (SMB) and value factor (HML) in addition to the market beta, which captures the cross-sectional variation in average stock returns. Moreover, Fama and French [1] found that the three-factor model can explain many regularities that are anomalous under the capital asset pricing model, including firm size, book-to-market (BM), past sales growth, long-run reversals, cash-flow-to-price, and earnings-toprice. However, Fama and French [2] claimed that their fivefactor model, which adds the profitability factor (RMW) and investment factor (CMA) to the three-factor model, is superior to their original three-factor model for US firms with new and longer data from July 1963 to December 2013. However, the search for factors that explain the cross section of expected stock returns has produced hundreds of potential candidates. A fundamental task facing the asset pricing field today is to bring more discipline to the proliferation of factors. In particular, a question that remains open is how to judge whether a new factor adds explanatory power for asset pricing, relative to the hundreds of factors the literature has so far produced?

In recent years, two more possible factors have been discovered, including momentum factor (MOM) and long memory factor, which is denoted by the Hurst parameter. Initially, Jegadeesh and Titman [3] proposed the momentum effect. In 1997, Carhart [4] observed the momentum effect of different maturities and extracted the momentum factor (MOM), which is the difference in the equal-weighted average return of the top 30\% stocks and the last 30\% stocks with a one-month lag in the past 11 months, to incorporate into the asset pricing model. The model explains the inertia of most fund performance. Ouyang and Fei [5] studied the applicability of the four-factor pricing model in China's stock market. They tested the four-factor asset pricing model with a six-month lagging momentum factor by region and industry and found that it has higher explanatory power than the three-factor and CAPM model.

In addition to the momentum effect, researchers have also conducted a lot of discussions on whether the time series of stock returns has the property of the long memory. 
Indeed, the Hurst exponent is often used to describe the long memory of a time series. The commonly used method for estimating the Hurst exponent is the R/S analysis method (Rescaled Range Analysis) proposed by Hurst [6]. Mandelbrot [7] first applied the R/S analysis method to securities market research. However, some scholars [8-10] have shown that when there is short-term memory in the time series, the results obtained by R/S analysis are biased. Lo [9] proposed a revised R/S analysis method, but Teverovasky et al. [11] believed that the revised R/S still has a big flaw because the method must be selected for parameters, and improper selection of parameters often results in large deviations. As far as we know, the commonly used nonparametric estimation method is log-periodogram regression. Its advantage is that the algorithm is relatively simple, but the accuracy and stability are poor. To overcome this obstacle, Robinson [12] proposed another semiparametric estimation method: local Whittle estimation method (LW). He proved that LW estimation is better than the log-periodogram regression method despite the need for numerical optimization. The detrended fluctuation analysis (DFA) method is a scale index method proposed by Peng et al. [13] based on DNA mechanism, which is used to analyze the long-range correlation of time series. This method is mainly to remove the local trend of the data on different time scales, but for a time series, if there is no trend and the specific form of the trend, there will be certain limitations. In addition, the DFA method and the R/S analysis method have a common defectinsufficient accuracy when the time series length is too short. Later, some algorithms dedicated to improving the estimation accuracy appeared gradually to be more effectively applied to the analysis of financial time series, including Quasi Maximum Likelihood (QML) analysis, Generalized Hurst Exponent (GHE), wavelet analysis, Centered Moving Average (CMA), multifractal detrended fluctuation analysis (MFDFA), a nonlinear tool similar with the Lyapunov exponent, geometric method-based procedures (GM), and fractal dimension algorithms (FD). The disadvantage of the maximum likelihood estimation method is weak consistency. The wavelet transform in the wavelet analysis method involves the selection of the fundamental wavelet function. If the selection is improper, the analysis result will be greatly biased. The CMA method has better stability when $n$ is small. Vitanov et al. [14] introduced the estimation method of Hurst exponent by MFDFA and used methods such as Lyapunov exponent and PCA to estimate the chaos of the system and compress the dimensions.

Researchers not only study the differences of Hurst exponent estimation methods but also incorporate the Hurst exponent's long memory interpretation of time series into the factor model and compare it with the momentum factor. For example, semiparametric estimation approaches involve the celebrated the R/S statistic introduced by Hurst [6]; the parameter estimation method includes the exact maximum likelihood estimation proposed by Beran [15]; Whittle maximum likelihood estimation provided by Fox and Taqqu [16] and Dahlhaus [17]; the quadratic variations approach proposed by Guyon and Leon [18] and Istas and Lang [19]; the modified R/S statistic provided by Lo [9]; the Higuchi's method (see, for example, Higuchi [20]); the detrended fluctuation analysis provided by Peng et al. [13]; the log-periodogram regression method proposed by Geweke and Porter-Hudak [21] and Robinson [22]; and the local Whittle method developed by Robinson [12]; Velasco [23]; Phillips and Shimotsu [24]; Shimotsu and Phillips [25]; Bardet and Kammoun [26]; and Shimotsu [27]. Nonparametric estimation includes the increment ratio method proposed in Surgailis et al. [28] and extended in Bardet and Surgailis [29]; the wavelet based method provided by Bardet and Kammoun [26]. López-García et al. [30] first analyzed the explanatory power of five-factor model on U.S. stock returns, and they introduced the fractal dimension algorithm (FD method), compared the Hurst exponent calculated by the FD method with the momentum factor, and pointed out the superiority of the Hurst exponent over the momentum factor in model interpretation. However, this paper based on Fama-French three-factor model only uses the FD method to calculate the Hurst exponent and has no robustness test of the Hurst estimation method. In this paper, we will analyze the explanatory power of the Hurst exponent factor based on the Fama and French five-factor model and estimate Hurst exponent by two methods to test the results for robustness.

For this purpose, we first use five factors to analyze the explanatory power of China's A-share stocks and establish a factor model that includes momentum factor and the Hurst exponent estimated by two methods. Then, we will fully compare the performance of Hurst exponent and momentum factor on model improvement and test the momentum effect and long memory of the time series in the Chinese capital market. In order to further explore the robustness of the results, we will use two popular methods to estimate the Hurst exponent, which are based on least squares method by Berzin et al. [31].

The paper is organized as follows. Section 2 introduces the five-factor model as proposed by Fama and French [2] and explains two-parameter methods for the Hurst parameter and momentum factor. Section 3 provides several empirical applications of the procedure and explores the robustness of the results. Section 4 gives the concluding remarks.

\section{Five-Factor Model and Hurst Exponent}

CAPM model is a classical model which describes stock returns as risk-free rate plus market premium risk return as follows:

$$
R_{i t}-R_{f t}=a_{i}+b_{i}\left(\mathrm{Mkt}_{t}-R_{f t}\right)+e_{i t},
$$

where $R_{i t}$ is the return on security $i$ for period $t, R_{f t}$ is the risk-free return, and $\mathrm{Mkt}_{t}$ is the difference between $R_{i t}$.

From the empirical evidence on U.S. stocks and the applications of CAPM, Fama and French $[1,32]$ proposed an extension of (1) by introducing two new factors and capturing patterns associated with the size and value versus growth stocks. The three-factor empirical asset pricing model is defined then as follows: 


$$
R_{i t}-R_{f t}=a_{i}+b_{i} \mathrm{Mkt}_{t}+s_{i} \mathrm{SMB}_{t}+h_{i} \mathrm{HML}_{t}+e_{i t},
$$

where $\mathrm{SMB}_{t}$ is the returns on a diversified portfolio of small stocks minus the returns on a diversified portfolio of big stocks and $\mathrm{HML}_{t}$ is the difference between the returns on diversified portfolios of high book-to-market and low bookto-market stocks.

Fama and French [2] introduced a five-factor asset pricing model that adds the profitability and investment factors to the three-factor model of Fama and French [32] as follows:

$$
\begin{aligned}
R_{i t}-R_{f t}= & a_{i}+b_{i} \mathrm{Mkt}_{t}+s_{i} \mathrm{SMB}_{t}+h_{i} \mathrm{HML}_{t} \\
& +r_{i} \mathrm{RMW}_{t}+c_{i} \mathrm{CMA}_{t}+e_{i t},
\end{aligned}
$$

where $\mathrm{RMW}_{t}$ is the difference between the returns on diversified portfolios of stocks with robust and weak profitability correspondingly, $\mathrm{CMA}_{t}$ is the difference between the returns on diversified portfolios of the stocks of companies with low and with high investment practices, and $e_{i t}$ is a zero-mean residual.

Long memory in economics and finance has attached a great attention since a ground-breaking work of Mandelbrot and Van Ness [33]. In fact, the long memory property of time series means a significant dependence between very distant observations and a pole in the neighborhood of the zero frequency of their spectrum. When stock market returns have the property of long memory, the Efficient Market Hypothesis is not confirmed. In this case, the distribution of the stock return has fat tails and is persistent. Thus, stock returns are highly correlated, and there is black noise and a trend in the market. Some early studies in long memory process in finance include the studies by Hurst [6]; Mandelbrot and Wallis [34]; and Lo [9]. To consider the long memory fact, we should estimate the Hurst parameter. In fact, there exists a vast literature that describes different methods for estimating the Hurst parameter of the $\mathrm{fBm}$.

In this paper, we use two strongly consistent and asymptotically normal estimators of Berzin et al. [31].

Let $\quad X_{t}=\log \left(\sigma_{t}\right), n \in \mathbb{N}^{+}-1, k \in \mathbb{R}^{+}$, and $M_{k}(n)=(1 / n-1) \sum_{i=0}^{n-k}\left(X_{(i+2) \Delta}-2 X_{(i+1) \Delta}+X_{i \Delta}\right)^{k}$.

For $i=1, \ldots, \ell$, Berzin et al. [31] introduced the following least squares estimator $H_{k}$ of $H$ as

$$
\widehat{H}_{k}=-\frac{1}{k} \sum_{i=1}^{\ell} z_{i} \log \left(M_{k}\left(n_{i}\right)\right) \text {, }
$$

where $n_{i}=r_{i} n, r_{i} \in \mathbb{N}^{*}, i=1, \ldots, \ell$ and $z_{i}=\left(y_{i} / \sum_{i=1}^{l} y_{i}^{2}\right)$ and $y_{i}=\log \left(r_{i}\right)-(1 / \ell)\left(\sum_{i=1}^{\ell} \log \left(r_{i}\right)\right)$. Moreover, let $M_{\log }(n)=(1 /(n-1)) \sum_{i=0}^{n-2} \log \left(\left|X_{(i+2) \Delta}-2 X_{(i+1) \Delta}+X_{i \Delta}\right|\right)$. Berzin et al. [31] introduced another least squares estimator $\widetilde{H}_{\log }$ of $H$ as follows:

$$
\widetilde{H}_{\log }=-\sum_{i=1}^{\ell} z_{i} M_{\log }\left(n_{i}\right) .
$$

From Remark 3.12 and Remark 3.15 of Berzin et al. [31], we can state the following asymptotic theory.

Corollary 1. The estimator $\widehat{H}_{k}$ is an asymptotically unbiased strongly consistent estimator of $H$, and the estimator $\widetilde{H}_{\log }$ is unbiased weakly consistent estimator of $\mathrm{H}$. Furthermore, for $k=2 r_{i}=2^{i-1}$ and $i=1, \ldots, \ell$, we have

$$
\begin{gathered}
\sqrt{n}\left(\widehat{H}_{k}-H\right) \stackrel{d}{\longrightarrow} \mathcal{N}\left(0, \sigma_{\hat{H}_{k}}^{2}\right), \\
\sqrt{n}\left(\widehat{H}_{\log }-H\right) \stackrel{d}{\longrightarrow} \mathcal{N}\left(0, \sigma_{\widetilde{H}_{\log }^{2}}^{2}\right),
\end{gathered}
$$

where

$$
\begin{aligned}
\sigma_{\widehat{H}_{k}}^{2}= & \left(\frac{6}{\log (2)}\right)^{2} \frac{1}{\ell^{2}\left(\ell^{2}-1\right)^{2}} 2\left(\sum_{i<j ; i, j=1}^{\ell} 2^{-j}(2 i-(\ell+1))(2 j-(\ell+1)) \times \sum_{r=-\infty}^{+\infty} \rho_{1,2 j-i}^{2}(r)+\sum_{i=1}^{\ell} 2^{-i}(2 i-(\ell+1))^{2} \sum_{r=-\infty}^{+\infty} \rho_{H}^{2}(r)\right), \\
\sigma_{\widetilde{H}_{\log }}^{2}= & \left(\frac{3}{\log (2)}\right)^{2} \frac{1}{\ell^{2}\left(\ell^{2}-1\right)^{2}}\left(2 \sum_{i<j ; i, j=1}^{\ell} 2^{-j+1}(2 i-(\ell+1))(2 j-(\ell+1))\right) \times \sum_{p=1}^{+\infty}(2 p) !\left(\frac{1}{p(2 p-1) ! !}\right)^{2} \sum_{r=-\infty}^{+\infty} \rho_{1,2 j-i}^{2 p}(r) \\
& +\sum_{i=1}^{\ell} 2^{-i+1}\left(2 i-(\ell+1)^{2}\right) \times \sum_{p=1}^{+\infty}(2 p) !\left(\frac{1}{p(2 p-1) ! !}\right)^{2} \sum_{r=-\infty}^{+\infty} \rho_{H}^{2 p}(r), \\
\rho_{b, c}(x)= & \frac{1}{2\left(4-2^{2 H}\right)}(b c)^{-H}\left[-|x|^{2 H}+2|x-b|^{2 H}-|x-2 b|^{2 H}+2|x+c|^{2 H}-4|x+c-b|^{2 H}\right. \\
& \left.+2|x+c-2 b|^{2 H}-|x+2 c|^{2 H}+2|x+2 c-b|^{2 H}-|x+2 c-2 b|^{2 H}\right], \\
\rho_{H}(x)= & \frac{-6|x|^{2 H}+4|x+1|^{2 H}-|x+2|^{2 H}-|x-2|^{2 H}+4|x-1|^{2 H}}{2\left(4-2^{2 H}\right)} .
\end{aligned}
$$


Because of the price limits policy in Chinese A-Share Market, in this paper, the momentum factor is compiled as follows:

$$
\mathrm{MOM}=\frac{X_{i+k}}{X_{i}}, \quad i=1,2,3, \ldots, n-k,
$$

where $x$ is the stock price and $K$ is the momentum factor parameter, usually 12 months which will be adjusted for robust test later.

\section{Empirical Application}

3.1. Five-Factor Model in Chinese Stock Market. We are going to examine the performance of the Fama-French five-factor model in Chinese stock market and analyze the performance of the Hurst factor and MOM in the factor model. The stock data in this study are daily data from January 2010 to July 2020. By sampling from each month, the monthly data of the time period are obtained. The financial data needed mainly come from the quarterly financial reports of listed companies. Since listed companies announce their financial reports at different times, there are always differences in the financial data collected at the end of each month in the A-share market. Hurst exponent and momentum factor are calculated based on monthly stock price data.

Similar to Fama and French [2], the four factors of SMB, HML, RMW, and CMA in the five-factor model are calculated based on the grouping of financial data on the monthly return rate of stocks. Then, we combine the monthly return rate of the market index with the return rate of the four factors to obtain the final value of the five factors in the month, cycle the calculation of the five factors every month, and finally get the five-factor data from February 2010 to June 2020 .

To study which factor in the five-factor model is more significant to explain stock returns, we use a combination of five factors as explanatory variables to construct a regression model. Five factors can form $C_{5}^{1}+C_{5}^{2}+C_{5}^{3}+C_{5}^{4}+C_{5}^{5}$ kinds of combinations; that is, 31 kinds of combinations can be formed. The Akaike [35] test (AIC) is used to select the optimal model; that is, when the AIC is the smallest, the model is regarded as the optimal model.

For all A shares, ignoring the fact that data errors cannot be regressed, the mean of $r$ square of the regression is 0.4002 . Table 1 reports the proportion of the five factors in the optimal model of each stock. The MKT is the factor with the highest proportion, which means that MKT has a universal explanatory power for A-share returns. RMA and SMB are also very important, accounting for close to 50\%, which means the company's profit fundamentals have a greater impact on stock returns of A shares and small market value effect is common. CMA performances are the weakest, which may be related to the large amount of data in the A-share financial statements.

3.2. Seven-Factor Model. The calculation of Hurst exponent and momentum factor both needs to determine a time series length. In this paper, the length of 12 month is selected for a
TABLE 1: The proportion of the five factors in the optimal model of each stock.

\begin{tabular}{lccccc}
\hline Factor & MKT & SMB & HML & RMW & CMA \\
\hline The proportion & 0.9091 & 0.4736 & 0.3824 & 0.5110 & 0.0418 \\
\hline
\end{tabular}

preliminary study, and then the parameter will be changed for a systematic study.

The momentum factor and the Hurst exponent, which are calculated by the $\widehat{H}_{k}$ algorithm and the $\widehat{H}_{\log }$ algorithm, are added into the five-factor model to form a seven-factor model.

Seven factors can form $C_{7}^{1}+C_{7}^{2}+C_{7}^{3}+C_{7}^{4}+C_{7}^{5}+C_{7}^{6}+$ $C_{7}^{7}$ kinds of combinations. The AIC criterion is still used for model selection. For all A shares, using the Hurst exponent calculated by the $\widehat{H}_{k}$ algorithm and the $\widehat{H}_{\log }$ algorithm, the mean of $r$ square is 0.4731 and 0.4729 , respectively.

The two different algorithms have no difference in the improvement of $r$ square, which is maintained at about $47 \%$ and has an increase of $7 \%$ relative to the five-factor model.

The proportion of the seven factors in the optimal model is shown in Table 2 . Using the $\widehat{H}_{k}$ and $\widehat{H}_{\log }$ algorithm to calculate Hurst exponent, the market factor MKT is the most significant, while MOM's proportion is second only to the market factor. RMW and SMB take a proportion about $50 \%$, and the result is consistent with the five-factor model, which shows that the newly added MOM and Hurst exponent have a certain degree of substitution to the five-factor model. The Hurst exponent also has a certain effect, and it often appears in the model at the same time as MOM.

From Table 2, we can find different Hurst exponent performance about the same, accounting for roughly $23 \%$. The proportions of the other 6 factors have not changed much, indicating that different algorithms of Hurst exponent have no substitute influence on other factors.

From Table 3, we check the cross effect of $H$ and MOM. Firstly, we can conclude that both $H$ and MOM are very important factors because there is a very low percent (roughly 9\%) of models which does not contain neither $\mathrm{H}$ nor MOM. Secondly, this shows that although these two factors portray the trend performance, they are complementary to each other, rather than substituting previously guessed because the percent of " $H$ and MOM" is as high as about $20 \%$.

3.3. Portfolio Factor Analysis. In order to see if our result above is stable, in this section, we will use random portfolio which consists of 10 and 30 stocks, respectively, to run the seven-factor model and check the result for consistency with that in Section 3.2.

The mean of $r$ square of 10,000 random portfolios of 10 stocks is 0.781194 for $\widehat{H}_{k}$ and 0.777463 for $\widehat{H}_{\log }$. This result is higher than the $r$ square of the single stock model. And Table 4 shows a higher percent of presence of factors. This is because the diversity of 10 stock portfolios lowers the unsystematic risk and improves the explanatory power of seven factors. 
TABLE 2: The proportion of the seven factors in the optimal model.

\begin{tabular}{lccccccc}
\hline Factor & MKT & SMB & HML & RMW & CMA & MOM & Hurst \\
\hline$\widehat{H}_{k}$ & 0.940152 & 0.485249 & 0.329306 & 0.505198 & 0.045518 & 0.876651 & 0.220849 \\
$\widehat{H}_{\log }$ & 0.938465 & 0.482719 & 0.330149 & 0.507165 & 0.04608 & 0.87918 & 0.239112 \\
\hline
\end{tabular}

TABLe 3: Percent of presence in the models of factors $H$ and MOM.

\begin{tabular}{lcccc}
\hline Factor & No one & $H$ but no MOM & MOM but no $H$ & \\
\hline$\widehat{H}_{k}$ & 0.090475 & 0.030346 & 0.670413 & 0.208767 \\
$\widehat{H}_{\log }$ & 0.094409 & 0.028941 & 0.684743 & 0.191908 \\
\hline
\end{tabular}

TABle 4: Percent of presence in the models of each factor for 10,000 random portfolios of 10 stocks.

\begin{tabular}{lccccccc}
\hline Factor & MKT & SMB & HML & RMW & CMA & MOM & Hurst \\
\hline$\widehat{H}_{k}$ & 0.972744 & 0.552933 & 0.330231 & 0.525843 & 0.051853 & 0.347017 & 0.280372 \\
$\widehat{H}_{\log }$ & 0.972114 & 0.554504 & 0.319419 & 0.524421 & 0.052222 & 0.355248 & 0.272773 \\
\hline
\end{tabular}

TABle 5: Percent of presence in the models of each factor for 10,000 random portfolios of 30 stocks.

\begin{tabular}{lccccccc}
\hline Factor & MKT & SMB & HML & RMW & CMA & MOM & Hurst \\
\hline$\widehat{H}_{k}$ & 0.990253 & 0.50288 & 0.393886 & 0.682322 & 0.100576 & 0.473638 \\
$\widehat{H}_{\log }$ & 0.99359 & 0.516026 & 0.409341 & 0.689103 & 0.091117 & 0.462454 & 0.391026 \\
\hline
\end{tabular}

TABLE 6: Mean of $r$ square under different parameters and Hurst exponent algorithms.

\begin{tabular}{lccc}
\hline Algorithm/parameter & 12 months & 24 months & 36 months \\
$\widehat{H}_{k}$ & 0.4731 & 0.472543 & 0.490673 \\
$\widehat{H}_{\log }$ & 0.4729 & 0.471874 & 0.508291 \\
\hline
\end{tabular}

TABLE 7: The proportion of each factor under different Hurst exponent algorithms.

\begin{tabular}{|c|c|c|c|c|c|c|c|}
\hline \multicolumn{8}{|c|}{ Panel A: Time parameter equals 12 months } \\
\hline Factor & MKT & SMB & HML & RMW & CMA & MOM & Hurst \\
\hline$\widehat{H}_{k}$ & 0.938466 & 0.48272 & 0.330149 & 0.507165 & 0.04608 & 0.87918 & 0.239112 \\
\hline$\widehat{H}_{\log }$ & 0.940152 & 0.485249 & 0.329306 & 0.505198 & 0.045518 & 0.876651 & 0.220849 \\
\hline \multicolumn{8}{|c|}{ Panel B: Time parameter equals 24 months } \\
\hline Factor & MKT & SMB & HML & RMW & CMA & MOM & Hurst \\
\hline$\widehat{H}_{k}$ & 0.934807 & 0.518141 & 0.374717 & 0.47619 & 0.061791 & 0.617063 & 0.268141 \\
\hline$\widehat{H}_{\log }$ & 0.933107 & 0.516156 & 0.376701 & 0.474206 & 0.060374 & 0.61763 & 0.25085 \\
\hline \multicolumn{8}{|c|}{ Panel C: Time parameter equals 36 months } \\
\hline Algorithm/factor & MKT & SMB & HML & RMW & CMA & MOM & Hurst \\
\hline$\widehat{H}_{k}$ & 0.940908 & 0.536095 & 0.401157 & 0.490405 & 0.088943 & 0.524825 & 0.271703 \\
\hline$\widehat{H}_{\log }^{n}$ & 0.921729 & 0.527268 & 0.406329 & 0.494207 & 0.088726 & 0.526137 & 0.291325 \\
\hline
\end{tabular}

Table 5 shows the result of 10,000 random portfolios of 30 stocks. The mean of $r$ square of 10,000 random portfolios of 30 stocks is 0.899578 for $\widehat{H}_{k}$ and 0.902907 for $\widehat{H}_{\log }$. Moreover, percent of presence of factor is even higher than that in Table 4. This result enhances the conclusion of the effect of diversity.
3.4. Robustness Test. Next, we will change the parameters of the momentum factor and the Hurst exponent to make a systematic comparison and observe whether the parameter changes have a significant impact on the results. The parameters are 12 months, 24 months, and 36 months. 
Table 6 shows the mean value of $r$ square of the sevenfactor model of the 2 Hurst exponent under different parameters. The mean value of $r$ square obtained by different time parameters under the same algorithm has little change. Overall, the mean value of $r$ square fluctuates not much when parameters vary, swinging between $47 \%-50 \%$.

Table 7 summarizes the proportion of each factor in the seven-factor model under different Hurst exponent algorithms. Among all the factors, the market factor accounts for the highest proportion, maintaining at around 93\%, which means that the market factor is undoubtedly the most explanatory factor in the Chinese A-share market. The MOM also accounts for a relatively high proportion, but the proportion gradually decreases as the time parameter increases, which means that the momentum effect usually has a large explanatory power in the short term, and the explanatory power gradually declines with the increase in time. SMB, HML, and RMW also have good explanatory power, and the proportions are relatively stable. The Hurst exponent gradually stabilizes with the increase in parameters, maintaining at about $27 \%$, which indicates Hurst exponent reflects a long-term trend. MOM captures more short-term trends, while Hurst exponent captures long-term trends. The two trends often appear in the same model instead of replacing each other.

\section{Conclusions}

In this paper, we first examine the performance of the Fama-French five-factor model in the Chinese A-share market. Choosing the AIC criterion as the criterion for model selection, we get the average value of $r$ square in the five-factor model, which is equal to 0.4002 . The most efficient factor is the market factor, and CMA performances are the weakest.

Then, we compile two kinds of Hurst exponents and add Hurst exponent and MOM to construct a seven-factor model. When the time parameter is 12 months, the mean value of $r$ square is about $47 \%$, which is $7 \%$ higher than that of the five-factor model. In terms of the explanatory power of each factor, the market factor is still the strongest (about 93\%), and the newly added MOM also has a strong explanatory power (about 87\%), and the SMB, HML, and RMW also have a certain degree efficiency, and the weakest is still CMA. The Hurst exponent has a strong explanatory power (about 23\%) and is complementary to MOM to a certain extent.

Finally, we study the sensitivity of time parameters. Set the time parameter to 12 months, 24 months, and 36 months, calculate MOM and Hurst exponent, and screen the seven-factor model. We find that the explanatory power of MOM gradually decreases with the increase in the parameters, and the Hurst exponent stabilizes at about $27 \%$ as the parameters increase, which explains that the Hurst exponent and MOM have complementary effects. MOM explains the short-term trend, while the Hurst exponent explains the long-term trend. The proportions of other factors are consistent with the previous model, and the difference between the two Hurst algorithms is not obvious.

\section{Data Availability}

The data used to support the findings of this study are available from the corresponding author upon request.

\section{Conflicts of Interest}

The authors declare that there are no conflicts of interest regarding the publication of this paper.

\section{Acknowledgments}

This study was supported by the Humanities and Social Sciences Research and Planning Fund of the Ministry of Education of China (Grant no. 20YJA630053).

\section{References}

[1] E. F. Fama and K. R. French, "The CAPM is wanted, dead or alive,” Journal of Finance, vol. 51, no. 5, pp. 1947-1958, 1996.

[2] E. F. Fama and K. R. French, "A five-factor asset pricing model," Journal of Financial Economics, vol. 116, no. 1, pp. 1-22, 2015.

[3] N. Jegadeesh and S. Titman, "Returns to buying winners and selling losers: implications for stock market efficiency," The Journal of Finance, vol. 48, no. 1, pp. 65-91, 1993.

[4] M. M. Carhart, "On persistence in mutual fund performance," Journal of Finance, vol. 52, no. 1, pp. 57-82, 1997.

[5] Z. Ouyang and L. Fei, "The applicability of the four-factor asset pricing model in Chinese stock market," Journal of Financial Economics, vol. 31, no. 2, pp. 84-96, 2016, in Chinese.

[6] H. E. Hurst, "Long-term storage capacity of reservoirs," Transactions of the American Society of Civil Engineers, vol. 116, pp. 770-808, 1951.

[7] B. Mandelbrot, "The variation of certain speculative prices," Journal of Business, vol. 36, no. 4, pp. 394-419, 1963.

[8] M. Couillard and M. Davison, "A comment on measuring the Hurst exponent of financial time series," Physica A: Statistical Mechanics and Its Applications, vol. 348, pp. 404-418, 2005.

[9] A. W. Lo, "Long-term memory in stock market prices," Econometrica, vol. 59, no. 5, pp. 1279-1313, 1991.

[10] M. A. Sánchez Granero, J. E. Trinidad Segovia, and J. García Pérez, "Some comments on Hurst exponent and the long memory processes on capital markets," Physica A: Statistical Mechanics and Its Applications, vol. 387, no. 22, pp. 55435551, 2008.

[11] V. Teverovsky, M. S. Taqqu, and W. Willinger, "A critical look at Lo's modified R/S statistic," Journal of Statistical Planning and Inference, vol. 80, no. 1-2, pp. 211-227, 1999.

[12] P. M. Robinson, "Gaussian semiparametric estimation of long range dependence," Annals of Stats, vol. 23, no. 5, pp. 1630-1661, 1995.

[13] C. K. Peng, S. V. Buldyrev, S. Havlin, M. Simons, H. E. Stanley, and A. L. Goldberger, "Mosaic organization of DNA nucleotides," Physical Review. E, Statal Physics, Plasmas, Fluids, and Related Interdiplinary Topics, vol. 49, no. 2, pp. 1685-1689, 1994.

[14] N. K. Vitanov, N. P. Hoffmann, and B. Wernitz, "Nonlinear time series analysis of vibration data from a friction brake: SSA, PCA, and MFDFA. Chaos," Solitons \& Fractals, vol. 69, pp. 90-99, 2014. 
[15] J. Beran, Statistics for Long-Memory Processes, CRC Press, New York, NY, USA, 1994.

[16] R. Fox and M. S. Taqqu, "Large-sample properties of parameter estimates for strongly dependent stationary Gaussian time series," Annals of Statistics, vol. 14, no. 2, pp. 517-532, 1986.

[17] R. Dahlhaus, "Efficient parameter estimation for self-similar processes," Annals of Statistics, vol. 17, no. 4, pp. 1749-1766, 1989.

[18] X. Guyon and J. León, "Convergence en loi des H-variations d'un processus gaussien stationnaire sur R," Annales de l'IHP Probabilités et Statistiques, vol. 25, no. 3, pp. 265-282, 1989.

[19] J. Istas and G. Lang, "Quadratic variations and estimation of the local holder index of a Gaussian process," Annales de l'Institut Henri Poincare (B) Probability and Statistics, vol. 33, pp. 407-436, 1997.

[20] T. Higuchi, "Approach to an irregular time series on the basis of the fractal theory," Physica D: Nonlinear Phenomena, vol. 31, no. 2, pp. 277-283, 1988.

[21] J. Geweke and S. Porter-Hudak, "The estimation and application of long memory time series models," Journal of Time Series Analysis, vol. 4, no. 4, pp. 221-238, 1983.

[22] P. M. Robinson, "Log-periodogram regression of time series with long range dependence," Annals of Statistics, vol. 23, no. 3, pp. 1048-1072, 1995.

[23] C. Velasco, "Gaussian semiparametric estimation of nonstationary time series," Journal of Time Series Analysis, vol. 20, no. 1, pp. 87-127, 1999.

[24] P. C. Phillips and K. Shimotsu, "Local whittle estimation in nonstationary and unit root cases," Annals of Statistics, vol. 32, no. 2, pp. 656-692, 2004.

[25] K. Shimotsu and P. C. Phillips, "Exact local whittle estimation of fractional integration," Annals of Statistics, vol. 33, no. 4, pp. 1890-1933, 2005.

[26] J.-M. Bardet and I. Kammoun, “Asymptotic properties of the detrended fluctuation analysis of long-range-dependent processes," IEEE Transactions on Information Theory, vol. 54, no. 5, pp. 2041-2052, 2008.

[27] K. Shimotsu, "Exact local whittle estimation of fractional integration with unknown mean and time trend," Econometric Theory, vol. 26, no. 2, pp. 501-540, 2010.

[28] D. Surgailis, G. Teyssiere, and M. Vaiciulis, "The increment ratio statistic," Journal of Multivariate Analysis, vol. 99, no. 3, pp. 510-541, 2008.

[29] J.-M. Bardet and D. Surgailis, "Measuring the roughness of random paths by increment ratios," Bernoulli, vol. 17, no. 2, pp. 749-780, 2011.

[30] M. N. López-García, J. E. Trinidad-Segovia, and M. SánchezGranero, "Extending the Fama and French model with a long term memory factor," European Journal of Operational Research, vol. 291, no. 2, pp. 421-426, 2021.

[31] C. Berzin, A. Latour, and J. R. León, Inference on the Hurst Parameter and the Variance of Diffusions Driven by Fractional Brownian Motion, Springer, Cham, Switzerland, 2014.

[32] E. F. Fama and K. R. French, "Common risk factors in returns on stocks and bonds," Journal of Financial Economics, vol. 33, no. 1, pp. 3-56, 1993.

[33] B. B. Mandelbrot and J. W. Van Ness, "Fractional Brownian motions, fractional noises and applications," SIAM Review, vol. 10, no. 4, pp. 422-437, 1968.

[34] B. B. Mandelbrot and J. R. Wallis, "Robustness of the rescaled range $\mathrm{R} / \mathrm{S}$ in the measurement of noncyclic long run statistical dependence," Water Resources Research, vol. 5, no. 5, pp. 967-988, 1969.
[35] H. Akaike, "A new look at the statistical model identification," IEEE Transactions on Automatic Control, vol. 19, no. 6, pp. 716-723, 1974. 